\title{
Scott is always simple
}

\author{
Antonino Salibra \\ salibra@dsi.unive.it \\ DAIS, Università Ca'Foscari Venezia \\ Via Torino 155, 30172 Venezia, Italy
}

\begin{abstract}
In this paper we give an outline of recent algebraic results concerning theories and models of the untyped lambda calculus.
\end{abstract}

\section{Introduction}

The lambda calculus was originally introduced by Church $[12,13]$ as a foundation for logic, where functions, instead of sets, were primitive, and it turned out to be consistent and successful as a tool for formalising all computable functions. The rise of computers and the development of programming languages gave a new development to its theoretical studies. The lambda calculus is the kernel of the functional programming paradigm, because its ordinary parameterbinding mechanism corresponds closely to parameter binding in many functional programming languages and to variable binding of quantifiers in logic.

Lambda calculus has been originally investigated by using mainly syntactical methods (see Barendregt's book [1]). At the beginning researchers have focused their interest on a limited number of equational extensions of lambda calculus, called $\lambda$-theories. They arise by syntactical or semantic considerations. Indeed, a $\lambda$-theory may correspond to a possible operational semantics of lambda calculus, as well as it may be induced by a model of lambda calculus through the kernel congruence relation of the interpretation function. The set of $\lambda$-theories is naturally equipped with a structure of complete lattice (see [1, Chapter 4$]$ ). The bottom element of this lattice is the least $\lambda$-theory $\lambda \beta$, while the top element is the inconsistent $\lambda$-theory. Although researchers have mainly focused their interest on a limited number of them, the lattice of $\lambda$-theories has a very rich and complex structure (see e.g. [1, 19,21]).

The lambda calculus, although its axioms are all in the form of equations, is not a genuine equational theory since the variable-binding properties of lambda abstraction prevent "variables" in lambda calculus from operating as real algebraic variables. There have been several attempts to reformulate the lambda calculus as a purely algebraic theory. The earliest, and best known, algebraic models are the combinatory algebras of Curry and Schönfinkel (see [15]). Although combinatory algebras do not keep the lambda notation, they have a simple purely equational characterisation and were used to provide an intrinsic first-order, but not equational, characterisation of the models of lambda calculus, as a special class of combinatory algebras called $\lambda$-models [1, Def. 5.2.7]. 
The connection between the syntax and the semantics of lambda calculus is established by the completeness theorem of lambda calculus: every $\lambda$-theory is the equational theory of some $\lambda$-model.

Semantical methods have been extensively investigated. After the first model, found by Scott [28] in 1969 in the category of complete lattices and Scott continuous functions, a large number of mathematical models for lambda calculus have been introduced in various categories of domains and were classified into semantics according to the nature of their representable functions, see e.g. [1, 3]. Scott continuous semantics [29] is given in the category whose objects are complete partial orders and morphisms are Scott continuous functions. Other semantics of lambda calculus were isolated by Berry [5] and Bucciarelli-Ehrhard [7]: Berry's stable semantics and Bucciarelli-Ehrhard's strongly stable semantics are refinements of the continuous semantics introduced to capture the notion of "sequential" Scott continuous function. All these semantics are structurally and equationally rich in the sense that it is possible to build up $2^{\aleph_{0}} \lambda$-models in each of them inducing pairwise distinct $\lambda$-theories (see $[17,18]$ ). Nevertheless, the above denotational semantics do not match all possible operational semantics of lambda calculus. We recall that a semantics of lambda calculus is equationally incomplete if there exists a $\lambda$-theory which is not the theory of any model in the semantics. In the nineties the problem of the equational incompleteness was positively solved by Honsell and Ronchi della Rocca [16] for Scott's continuous semantics, and by Bastonero and Gouy for Berry's stable semantics [2]. The proofs of the above results are syntactical and very difficult. In [26] the author has provided an algebraic and simple proof of the equational incompleteness of all semantics of lambda calculus that involve monotonicity with respect to some partial order and have a bottom element (including the incompleteness of the strongly stable semantics, which had been conjectured by Bastonero-Gouy and by Berline $[2,3])$.

The need of more abstract and sophisticated mathematical techniques in lambda calculus arises when we recognise the difficulty of the problems we handle, for example in order to investigate the structure of the lattice of $\lambda$-theories in itself and in connections with the theory of models. The author $[19,25,26]$ has launched at the end of the nineties a research program for exploring lambda calculus and combinatory logic using techniques of universal algebra. The remark that the lattice of $\lambda$-theories is isomorphic to the congruence lattice of the term algebra of the least $\lambda$-theory $\lambda \beta$ is the starting point for studying lambda calculus by universal algebraic methods, through the variety (i.e., equational class) generated by the term algebra of $\lambda \beta$. In [25] the author has shown that the variety generated by the term algebra of $\lambda \beta$ is axiomatised by the finite schema of identities characterising $\lambda$-abstraction algebras (see [23]). The variety of $\lambda$-abstraction algebras is an algebraic description of lambda calculus, which keeps the lambda notation and hence all the functional intuitions. In [25] it was shown that, for every variety of $\lambda$-abstraction algebras, there exists exactly one $\lambda$-theory whose term algebra generates the variety. Thus, the properties of a $\lambda$-theory can be studied by means of the variety of $\lambda$-abstraction algebras gener- 
ated by its term algebra. By applying these methods it was shown in [19] that the lattice of $\lambda$-theories satisfies a nontrivial implication in the language of lattices. It is open whether the lattice of $\lambda$-theories satisfies a nontrivial lattice identity (see [21, Section 4] for other results concerning the structure of the lattice of $\lambda$-theories).

Longstanding open problems of lambda calculus can be restated in terms of algebraic properties of varieties of $\lambda$-abstraction algebras or combinatory algebras. For example, the open problem of the order-incompleteness of lambda calculus, raised by Selinger (see [30]), asks for the existence of a $\lambda$-theory not arising as the equational theory of a non-trivially partially ordered model of lambda calculus. A partial answer to the order-incompleteness problem was obtained by the author in [26], where it is shown the existence of a $\lambda$-theory not arising as the equational theory of a non-trivially partially ordered model with a finite number of connected components. The order-incompleteness of lambda calculus is equivalent to the existence of an $n$-permutable variety of combinatory algebras for some natural number $n \geq 2$ (see the remark after Thm. 3.4 in [30]). Plotkin, Selinger and Simpson (see [30]) have shown that 2-permutability and 3-permutability are inconsistent with lambda calculus. The problem of $n$ permutability remains open for $n \geq 4$.

One of the milestones of modern algebra is the Stone representation theorem for Boolean algebras. This result was first generalised by Pierce [22] to commutative rings with unit and next by Comer [14] to the class of algebras with Boolean factor congruences. Comer's generalisation of Stone representation theorem also holds for combinatory algebras (see [21]): any combinatory algebra is isomorphic to a weak Boolean product of directly indecomposable algebras (i.e., algebras which cannot be decomposed as the Cartesian product of two other non-trivial algebras). The proof of the representation theorem is based on the fact that the directly indecomposable combinatory algebras constitute a universal class and that every combinatory algebra contains a Boolean algebra of central elements (introduced by Vaggione [31] in universal algebra). These elements define a direct decomposition of the algebra as the Cartesian product of two other algebras, just like idempotent elements in rings. This approach to central elements can be developed in the more general context of Church algebras, which were introduced in [20] to equationally axiomatise the "if-then-else" construct of programming.

The Stone representation theorem can be roughly summarised as follows: the directly indecomposable combinatory algebras are the 'building blocks' of the variety of combinatory algebras. The notion of directly indecomposable combinatory algebra appears to be so relevant that it is even interesting to speak of the "indecomposable semantics" to denote the class of models of $\lambda$-calculus which are directly indecomposable as combinatory algebras. This semantics encompasses the Scott continuous, stable and strongly stable semantics and was shown incomplete in [21].

The paper is organised as follows. In Section 2 we review the basic definition of lambda calculus and universal algebra. Church algebras are presented in Sec- 
tion 3. We provide the algebraic incompleteness theorem in Section 4. The last section is devoted to the order-incompleteness problem of $\lambda$-calculus.

\section{Preliminaries}

\subsection{Lambda calculus}

With regard to the $\lambda$-calculus we follow the notation and terminology of [1]. $\Lambda$ and $\Lambda^{\circ}$ are, respectively, the set of $\lambda$-terms and of closed $\lambda$-terms. $\Omega$ denotes the looping term $(\lambda x . x x)(\lambda x . x x)$.

We denote $\alpha \beta$-conversion by $\lambda \beta$ and $\alpha \beta \eta$-conversion by $\lambda \beta \eta$. A $\lambda$-theory is a congruence on $\Lambda$ (with respect to the operators of abstraction and application) which contains $\lambda \beta$. A $\lambda$-theory is consistent if it does not equate all $\lambda$-terms, inconsistent otherwise. The set of $\lambda$-theories constitutes a complete lattice w.r.t. intersection, whose top is the inconsistent $\lambda$-theory and whose bottom is the theory $\lambda \beta$. The $\lambda$-theory generated by a set $E$ of identities between $\lambda$-terms is the intersection of all $\lambda$-theories containing $E$.

There are two descriptions of what a model of $\lambda$-calculus is: the categorytheoretical and the algebraic one. The categorical notion of model is well-suited for constructing concrete models, while the algebraic one is rather used to understand global properties of models (constructions of new models out of existing ones, closure properties, etc.) and to obtain results about the structure of the lattice of $\lambda$-theories. In the remaining part of this section we define the algebraic notion of model.

Definition 1. An algebra $\mathbf{C}=(C, \cdot, \mathbf{k}, \mathbf{s})$, where $\cdot$ is a binary operation and $\mathbf{k}, \mathbf{s}$ are constants, is a combinatory algebra if it satisfies the identities $\mathbf{k} x y=x$ and $\mathbf{s} x y z=x z(y z)$ (as usual, the symbol ". is omitted and association is made on the left).

In the equational language of combinatory algebras we define $\mathbf{i}, \mathbf{1}$ and $\mathbf{1}_{n}$ as follows: $\mathbf{i} \equiv \mathbf{s k k} ; \mathbf{1} \equiv \mathbf{1}_{1} \equiv \mathbf{s}(\mathbf{k i})$ and $\mathbf{1}_{n+1} \equiv \mathbf{s}(\mathbf{k} \mathbf{1})\left(\mathbf{s}\left(\mathbf{k} \mathbf{1}_{n}\right)\right)$. Hence, every combinatory algebra satisfies $\mathbf{i} x=x$ and $\mathbf{1}_{n} x_{1} \ldots x_{n}=x_{1} \ldots x_{n}$.

A function $f: C \rightarrow C$ is representable in a combinatory algebra $\mathbf{C}$ if there exists an element $c \in C$ such that $c z=f(z)$ for all $z \in C$.

Two elements $x, y \in C$ are called extensionally equal if they represent the same function in $\mathbf{C}$. For example, the elements $x$ and $\mathbf{1} x$ are extensionally equal.

An environment is a function $\rho: \operatorname{Var} \rightarrow C$, where $\operatorname{Var}$ is the set of variables of $\lambda$-calculus. For every variable $x$ and $a \in C$ we denote by $\rho[x:=a]$ the environment $\rho^{\prime}$ which coincides with $\rho$, except on $x$, where $\rho^{\prime}$ takes the value $a$.

Given a combinatory algebra $\mathbf{C}$, the interpretation of a $\lambda$-term $M$ is defined by induction as follows, for every environment $\rho$ :

$$
|x|_{\rho}=\rho(x) ; \quad|M N|_{\rho}=|M|_{\rho} \cdot|N|_{\rho} ; \quad|\lambda x \cdot M|_{\rho}=\mathbf{1} \mathrm{m},
$$

where $m \in C$ is any element representing the function $a \in C \mapsto|M|_{\rho[x:=a]}$. The drawback of the previous definition is that it may happen that the function $a \in$ 
$C \mapsto|M|_{\rho[x:=a]}$ is not representable in $\mathbf{C}$. The axioms characterising $\lambda$-models were expressly chosen to make coherent the previous definition of interpretation.

Definition 2. A combinatory algebra $\mathbf{C}$ is called a $\lambda$-model if it satisfies the identities $\mathbf{1}_{2} \mathbf{k}=\mathbf{k}, \mathbf{1}_{3} \mathbf{s}=\mathbf{s}$ and the Meyer-Scott axiom:

$$
\forall x \forall y(\forall z(x z=y z) \Rightarrow 1 x=1 y)
$$

Here the combinator $\mathbf{1}$ is used as an inner choice operator. Indeed, given any $x \in C$, the element $\mathbf{1} x$ is in the same equivalence class as $x$ w.r.t. extensional equality; and, by Meyer-Scott axiom, $\mathbf{1} x=\mathbf{1} y$ for every $y$ extensionally equal to $x$. Thus, the set $Y$ of elements representing the function $a \in C \mapsto|M|_{\rho[x:=a]}$ admits $1 \mathrm{~m}$ as a canonical representative and this does not depend on the choice of $m \in Y$.

We write $\mathbf{C} \models M=N$ if $|M|_{\rho}=|N|_{\rho}$ for all environments $\rho$. A $\lambda$-model univocally induces a $\lambda$-theory through the kernel congruence relation of the interpretation function:

$$
T h(\mathbf{C})=\{(M, N) \in \Lambda \times \Lambda: \mathbf{C}=M=N\} .
$$

If $T$ is a $\lambda$-theory, the (open) term model of $T$ is the algebra $(\Lambda / T, \cdot, \mathbf{k}, \mathbf{s})$, where $t / T \cdot u / T=(t u) / T$ and $\mathbf{k}, \mathbf{s}$ are respectively the equivalence classes of $\lambda x y . x$ and $\lambda x y z . x z(y z)$ modulo $T$.

A partially ordered $\lambda$-model, a po-model for short, is a pair $(\mathbf{A}, \leq)$, where $\mathbf{A}$ is a $\lambda$-model and $\leq$ is a partial order on $A$ which makes the application operator of A monotone in both arguments. A po-model $(\mathbf{A}, \leq)$ is non-trivial if the partial order is not discrete, i.e., $a<b$ for some $a, b \in A$ (thus $A$ is not a singleton).

\subsection{Algebras}

With regard to Universal Algebra we follow the notation and terminology of [9]. A type $\nu$ is a set of operation symbols of finite arity. An algebra $\mathbf{A}$ of type $\nu$ is a tuple $\left(A, \sigma^{\mathbf{A}}\right)_{\sigma \in \nu}$, where, for every $\sigma \in \nu$ of arity $n, \sigma^{\mathbf{A}}$ is a function from $A^{n}$ into $A$.

Given two algebras $\mathbf{A}$ and $\mathbf{B}$ of type $\nu$, a homomorphism from $\mathbf{A}$ into $\mathbf{B}$ is a map $g: A \rightarrow B$ such that $g\left(\sigma^{\mathbf{A}}\left(a_{1}, \ldots, a_{n}\right)\right)=\sigma^{\mathbf{B}}\left(g\left(a_{1}\right), \ldots, g\left(a_{n}\right)\right)$ for each $n$-ary operation $\sigma \in \nu$ and for all $a_{i} \in A$. Two algebras $\mathbf{A}$ and $\mathbf{B}$ are isomorphic, and we write $\mathbf{A} \cong \mathbf{B}$, if there exists a bijective homomorphism from $\mathbf{A}$ into $\mathbf{B}$.

Given an algebra $\mathbf{A}$ of type $\nu$, a binary relation $\phi$ on $\mathbf{A}$ is compatible if for all $\sigma \in \nu$ of arity $n$, and for all $a_{i}, b_{i} \in A$ we have

$$
a_{1} \phi b_{1}, \ldots, a_{n} \phi b_{n} \rightarrow f^{\mathbf{A}}\left(a_{1}, \ldots, a_{n}\right) \phi f^{\mathbf{A}}\left(b_{1}, \ldots, b_{n}\right) .
$$

A compatible equivalence relation is called a congruence.

The kernel of a homomorphism $g: \mathbf{A} \rightarrow \mathbf{B}$ is the congruence $k e r(g)=$ $\left\{(a, b) \in A^{2}: g(a)=g(b)\right\}$. 
Given two congruences $\phi$ and $\psi$, we can form their relative product: $\psi \circ \phi=$ $\{(a, c):(\exists b \in A) a \phi b \psi c\}$. It is easy to check that $\psi \circ \phi$ is still a compatible relation, but not necessarily a congruence.

We denote by $\operatorname{Con}(\mathbf{A})$ the complete lattice of the congruences of $\mathbf{A}$, which is a sublattice of the lattice of the equivalence relations on $\mathbf{A}$. The meet $\phi \wedge \psi$ of two congruences $\phi$ and $\psi$ is their intersection, while their join is the least equivalence relation including $\phi \cup \psi$ :

$$
\phi \vee \psi=\bigcup_{n>0} \phi \circ^{n} \psi
$$

where $\psi \circ^{1} \phi=\psi$ and $\psi \circ^{n+1} \phi=\psi \circ\left(\phi \circ^{n} \psi\right)$ for $n>0$. The diagonal $\Delta_{\mathbf{A}}=$ $\{(a, a): a \in A\}$ is the bottom element of $\operatorname{Con}(\mathbf{A})$, while $\nabla_{\mathbf{A}}=A \times A$ is the top element.

$\mathbf{F}_{\nu}(X)$ is the absolutely free algebra of type $\nu$ over a set $X$ of generators. The elements of $\mathbf{F}_{\nu}(X)$ are called terms and are built up by induction: (i) every element of $X$ is a term; (ii) if $\sigma \in \nu$ is an operation symbol of arity $n$ and $p_{1}, \ldots, p_{n}$ are terms, then $\sigma\left(p_{1}, \ldots, p_{n}\right)$ is a term. The operations of $\mathbf{F}_{\nu}(X)$ are the syntactical operations of term construction.

Hereafter, we omit the index $\nu$ because all algebras will be always of type $\nu$.

If $\mathbf{A}$ is an algebra, then, for every map $\rho: X \rightarrow A$, there exists a unique homomorphism $\rho^{*}: \mathbf{F}(X) \rightarrow \mathbf{A}$ extending $\rho: \rho^{*}(x)=\rho(x)$ for every $x \in X$; $\rho^{*}\left(\sigma\left(p_{1}, \ldots, p_{n}\right)\right)=\sigma^{\mathbf{A}}\left(\rho^{*}\left(p_{1}\right), \ldots, \rho^{*}\left(p_{n}\right)\right)$.

For every term $p$, we define $p^{\mathbf{A}}: A^{X} \rightarrow A$ the map defined by $p^{\mathbf{A}}(\rho)=\rho^{*}(p)$. When $X=\left\{x_{1}, \ldots, x_{n}\right\}$ is a finite set of cardinality $n$, we define $p^{\mathbf{A}}: A^{n} \rightarrow A$ in the following equivalent way:

$$
p^{\mathbf{A}}\left(a_{1}, \ldots, a_{n}\right)=\rho^{*}(p), \text { where } \rho\left(x_{i}\right)=a_{i} \text { for } i=1, \ldots, n .
$$

$p^{\mathbf{A}}$ is called a term operation of $\mathbf{A}$.

An algebra $\mathbf{A}$ satisfies an equation $p=q(p, q \in F(X))$ if $p^{\mathbf{A}}=q^{\mathbf{A}}$. We write $\mathbf{A} \models p=q$ if $\mathbf{A}$ satisfies the equation $p=q$.

If $\mathbf{A}$ is an algebra, we denote by $E q_{X}(\mathbf{A})(E q(\mathbf{A})$ for short) the set of equations $p=q(p, q \in F(X))$ satisfied by $\mathbf{A}$.

If $\mathcal{K}$ is a class of algebras, then $E q(\mathcal{K})=\{p=q:(\forall \mathbf{A} \in \mathcal{K}) \mathbf{A} \models p=q\}$ is the set of equations satisfied by every algebra of $\mathcal{K}$.

A class $\mathcal{K}$ of algebras is (i) equational if it is axiomatised by a set of equations; (ii) a variety if it is closed under Cartesian products, subalgebras and homomorphic images. A class of algebras is equational iff it is a variety.

\subsection{The building blocks of algebras}

Direct products. The Cartesian product $\mathbf{B} \times \mathbf{C}$ of two algebras $\mathbf{B}$ and $\mathbf{C}$ of the same type has $B \times C$ as universe and operations defined as follows, for every $a_{i} \in B$ and $b_{i} \in C$ :

$$
\sigma^{\mathbf{B} \times \mathbf{C}}\left(\left\langle a_{1}, b_{1}\right\rangle, \ldots,\left\langle a_{n}, b_{n}\right\rangle\right)=\left\langle\sigma^{\mathbf{B}}\left(a_{1}, \ldots, a_{n}\right), \sigma^{\mathbf{C}}\left(b_{1}, \ldots, b_{n}\right)\right\rangle .
$$


Lemma 1. An algebra $\mathbf{A}$ is isomorphic to the Cartesian product $\mathbf{B} \times \mathbf{C}$ iff there exist two congruences $\theta, \bar{\theta} \in \operatorname{Con}(\mathbf{A})$ such that $\mathbf{B} \cong \mathbf{A} / \theta, \mathbf{C} \cong \mathbf{A} / \bar{\theta}, \theta \cap \bar{\theta}=\Delta_{\mathbf{A}}$ and $\theta \circ \bar{\theta}=\nabla_{\mathbf{A}}$.

Definition 3. A pair of congruences $\langle\theta, \bar{\theta}\rangle$ is called a pair of complementary factor congruences if $\theta \cap \bar{\theta}=\Delta, \theta \circ \bar{\theta}=\nabla$. A pair $\langle\theta, \bar{\theta}\rangle$ is trivial if either $\theta=\Delta$ or $\bar{\theta}=\Delta$.

A congruence $\theta$ is called a factor congruence if there exists $\bar{\theta}$ such that $(\theta, \bar{\theta})$ is a pair of complementary factor congruences. We denote by $F C(\mathbf{A})$ the set of factor congruence of $\mathbf{A}$. In general, $F C(\mathbf{A})$ is not a sublattice of $\mathrm{Con}(\mathbf{A})$.

Definition 4. An algebra $\mathbf{A}$ is directly indecomposable if it admits only the trivial pair of complementary factor congruences.

In other words, $\mathbf{A}$ is directly indecomposable if, and only if, it cannot be decomposed as a nontrivial Cartesian product. Then the directly indecomposable algebras are the building blocks for products.

Factors congruences and decomposition operators. Let $(\theta, \bar{\theta})$ be a pair of complementary factor congruences. Define a function $f_{\theta}: A \times A \rightarrow A$ as follows:

$$
f_{\theta}(a, b)=\text { the unique } c \text { such that } a \theta \bar{c} \bar{\theta} b \text {. }
$$

Proposition 1. The function $f_{\theta}$ satisfies the following conditions (for short, we write $f$ for $f_{\theta}$ ):

(D1) $f(x, x)=x$;

(D2) $f(x, f(y, z))=f(x, z)=f(f(x, y), z)$;

(D3) $f$ is a homomorphism from $\mathbf{A} \times \mathbf{A}$ into $\mathbf{A}$.

Definition 5. A function $f: A \times A \rightarrow A$ satisfying conditions (D1)-(D3) is called a decomposition operator.

Proposition 2. Given a decomposition operator $h: A \times A \rightarrow A$, the pair $\left(\theta_{h}, \bar{\theta}_{h}\right)$, defined by $a \theta_{h} b \Leftrightarrow h(a, b)=a ; \quad a \bar{\theta}_{h} b \Leftrightarrow h(a, b)=b$, is a pair of complementary factor congruences.

In conclusion, we have:

Proposition 3. There is a bijective correspondence between decomposition operators and factor congruences in such a way that

$$
\theta \mapsto f_{\theta} \mapsto \theta_{f_{\theta}}=\theta ; \quad h \mapsto \theta_{h} \mapsto f_{\theta_{h}}=h .
$$

There is always a battle to simplify complex objects. Decomposition operators are more suitable to be internalized within an algebra than pairs of complementary factor congruences. We are particularly interested in algebras, whose decomposition operators are term operations of the algebra. 
Subdirect and Boolean products. If $\mathbf{A}$ and $\mathbf{B}$ are algebras, then we write $\mathbf{A} \leq \mathbf{B}$ if there is an embedding (= injective homomorphism) from $\mathbf{A}$ into $\mathbf{B}$.

Definition 6. An algebra $\mathbf{A}$ is a subdirect product of an indexed family $\left(\mathbf{A}_{i}\right)_{i \in I}$ of algebras if $\mathbf{A} \leq \Pi_{i \in I} \mathbf{A}_{i}$ and $\pi_{i}(A)=A_{i}$ (where $\pi_{i}: A \rightarrow A_{i}$ is the projection in the $i$-coordinate).

A direct product is an example of subdirect product.

Lemma 2. Let $\theta_{i} \in \operatorname{Con}(\mathbf{A})$ be a family of congruences of an algebra $\mathbf{A}$. $\mathbf{A}$ is a subdirect product of the family $\left(\mathbf{A} / \theta_{i}\right)_{i \in I}$ (through the embedding $f(a)=\left(a / \theta_{i}\right.$ : $i \in I)$ ) if, and only if, $\cap_{i \in I} \theta_{i}=\Delta$.

Definition 7. An algebra is subdirectly irreducible (s.i., for short) if, for every representation $f: \mathbf{A} \rightarrow \Pi_{i \in I} \mathbf{A}_{i}$ as subdirect product, there exists $j$ such that $\pi_{j} \circ f: \mathbf{A} \rightarrow \mathbf{A}_{i}$ is an isomorphism.

Proposition 4. A is s.i. iff $\cap(\operatorname{Con}(\mathbf{A})-\{\Delta\}) \neq \Delta$ (in this case, $\cap(\operatorname{Con}(\mathbf{A})-$ $\{\Delta\})$ is called the monolith).

Theorem 1. (Birkhoff) Every algebra $\mathbf{A}$ is a subdirect product of s.i. algebras.

An algebra $\mathbf{A}$ is simple if $\operatorname{Con}(\mathbf{A})=\{\Delta, \nabla\}$. We have:

Simple $\subseteq$ Subdirectly Irreducible $\subseteq$ Directly Indecomposable.

Example 1. 1. Every two elements algebra is simple.

2. Every model of $\lambda$-calculus living in Scott continuous semantics is a simple combinatory algebra.

3. A vector space over a field is s.i. iff it is one-dimensional.

4. A Heyting algebra is s.i. iff there is a greatest element strictly below 1 .

5. Every algebra of cardinality a prime number $p$ is directly indecomposable.

6. The Stone representation theorem for Boolean algebras is a consequence of Theorem 1, because the algebra of truth values is the unique s.i. Boolean algebra.

Stone's representation theorem, perhaps the most distinctive result characterising Boolean algebras (or Boolean rings), can be generalised to a much larger class of algebras. The appropriate tool to attain this goal is the technique of Boolean products, which can be loosened to the notion of weak Boolean product to take care of somewhat less manageable cases. Pierce [22] proved that every commutative ring with unit is representable as a weak Boolean product of directly indecomposable rings. The technique of Boolean products underwent remarkable developments over the subsequent years (see e.g. [9, Ch. 4.8]), giving rise to further generalisations of Stone's theorem by Comer (covering the case of algebras with Boolean factor congruences [14]) and Vaggione [31].

Definition 8. $A$ (weak) Boolean product of a family $\left(\mathbf{A}_{i}\right)_{i \in I}$ of algebras is a subdirect product $\mathbf{A} \leq \prod_{i \in I} \mathbf{A}_{i}$, where $I$ can be endowed with a Boolean space topology such that: (i) the set $\left\{i \in I: a_{i}=b_{i}\right\}$ is (open) clopen for all $a, b \in A$, and (ii) if $a, b \in A$ and $N \subseteq I$ is clopen, then the element c, defined by $c_{i}=a_{i}$ for $i \in N$ and $c_{i}=b_{i}$ for $i \in I-N$, belongs to $A$. 


\section{Church algebras}

The key observation motivating the introduction of Church algebras [20] is that many algebras arising in completely different fields of mathematics, including Heyting algebras, rings with unit, combinatory algebras or $\lambda$-calculus, have a term operation $q$ satisfying the fundamental properties of the if-then-else connective: $q(1, x, y)=x$ and $q(0, x, y)=y$. As simple as they may appear, these properties are enough to yield rather strong algebraic results, which will be applied to $\lambda$-calculus in the next section.

Definition 9. We say that an algebra $\mathbf{A}$ is a Church algebra if it admits a ternary term operation $q(x, y, z)$ and two constants 0,1 such that the following identities are satisfied by $\mathbf{A}$ :

$$
q(1, x, y)=x ; \quad q(0, x, y)=y .
$$

A Church variety is a variety of Church algebras.

Example 2.

1. Rings with unit: $q(x, y, z)=x y+(1-x) z$;

2. Heyting algebras: $q(x, y, z)=(x \vee z) \wedge((x \rightarrow 0) \vee y)$;

3. Combinatory algebras: $q(x, y, z)=(x y) z, 1=\mathbf{k}$ and $0=\mathbf{s k}$;

4. Lambda calculus: $q(x, y, z)=(x y) z ; 1=\lambda x y . x$ and $0=\lambda x y . y$.

We denote by $\theta(a, b)$ the least congruence generated by the pair $(a, b)$.

Lemma 3. Let $\mathbf{A}$ be a Church algebra, $(\phi, \bar{\phi})$ be a pair of complementary factor congruences, and $e$ be the unique element such that $1 \phi e \bar{\phi} 0$. Then, we have:

(i) For every $a, b \in A, a \phi q(e, a, b) \bar{\phi} b$.

(ii) $\phi=\theta(1, e)$ and $\bar{\phi}=\theta(0, e)$.

(iii) The function $f_{\phi}(a, b)=q(e, a, b)$ is a decomposition operator on $\mathbf{A}$ such that $f_{\phi}(1,0)=e$.

In this definition we exploit an idea by Vaggione [31].

Definition 10. An element $e$ of a Church algebra $\mathbf{A}$ is central if $\theta(1, e)$ and $\theta(0, e)$ constitute a pair of complementary factor congruences of $\mathbf{A}$. $\mathrm{Ce}(A)$ denotes the set of central elements of the algebra $\mathbf{A}$.

Proposition 5. If $\mathbf{A}$ is a Church algebra and $e \in A$, then the following conditions are equivalent:

1. e is central;

2. For all $a, b, \bar{a}, \bar{b} \in A$ :

D1. $q(e, a, a)=a$

D2. $q(e, q(e, a, b), c)=q(e, a, c)=q(e, a, q(e, b, c))$

D3. $q(e, \sigma(\bar{a}), \sigma(\bar{b}))=\sigma\left(q\left(e, a_{1}, b_{1}\right), \ldots, q\left(e, a_{n}, b_{n}\right)\right) \quad$ (for every operation $\left.\sigma\right)$

D4. $q(e, 1,0)=e$. 
The partial ordering on $\mathrm{Ce}(A)$, defined by:

$$
e \leq d \text { if, and only if, } \theta(0, e) \subseteq \theta(0, d)
$$

is a Boolean ordering. The meet, join and complementation operations are internally representable, and 0,1 are respectively the bottom and top element of this ordering.

Theorem 2. Let $\mathbf{A}$ be a Church algebra.

(i) The set $F C(\mathbf{A})$ of factor congruences of $\mathbf{A}$ constitutes a Boolean sublattice of $\operatorname{Con}(\mathbf{A})$.

(ii) The algebra $\mathrm{Ce}(\mathbf{A})=(\mathrm{Ce}(A) ; \vee, \wedge, \neg, 0,1)$, where $x \wedge y=q(x, y, 0), x \vee y=$ $q(x, 1, y)$ and $\neg x=q(x, 0,1)$, is a Boolean algebra isomorphic to the Boolean algebra of factor congruences of $\mathbf{A}$.

\subsection{The Stone representation theorem}

A generic Church variety admits a weak Boolean product representation. The following theorem is a consequence of [9, Theorem 8.12].

Theorem 3. Let $\mathbf{A}$ be a Church algebra, $S$ be the Boolean space of maximal ideals of $\mathrm{Ce}(\mathbf{A})$ and $f: A \rightarrow \Pi_{I \in S} A / \theta_{I}$ be the map defined by

$$
f(a)=\left(a / \theta_{I}: I \in S\right),
$$

where $\theta_{I}=\bigcup_{e \in I} \theta(0, e)$. Then $f$ gives a weak Boolean representation of $\mathbf{A}$.

For the previous representation to be of some interest, we need to be in a position to provide additional information on its stalks. The following theorem is a consequence of [31, Theorem 8]. A new proof can be found in [27].

Theorem 4. Let $\mathcal{V}$ be a Church variety. Then, the following conditions are equivalent:

(i) For all $\mathbf{A} \in \mathcal{V}$, the stalks $\mathbf{A} / \theta_{I}$ (I $\in S$ maximal ideal) are directly indecomposable.

(ii) The class $\mathcal{V}_{D I}$ of directly indecomposable members of $\mathcal{V}$ is a universal class.

\section{The incompleteness theorem of lambda calculus}

The Stone representation theorem for combinatory algebras can be roughly summarised as follows: the directly indecomposable combinatory algebras are the "building blocks" in the variety of combinatory algebras. Then it is natural to investigate the class of models of $\lambda$-calculus, which are directly indecomposable as combinatory algebras (indecomposable semantics, for short).

In this section we show that the indecomposable semantics encompasses the Scott, stable and strongly stable semantics. In spite of this richness, we show that there exists a consistent $\lambda$-theory which is not the equational theory of an indecomposable model. The results in this section can be found in [21]. 


\subsection{Scott models are simple algebras}

After Scott, several models of $\lambda$-calculus have been defined by order theoretic methods and classified into "semantics" according to the nature of their representable functions (see [3], for a survey on these semantics).

The Scott-continuous semantics corresponds to the class of $\lambda$-models having cpo's (complete partial orders) as underlying sets and representing all Scott continuous functions.

The stable semantics (Berry [5]) and the strongly stable semantics (BucciarelliEhrhard [7]) are refinements of the Scott-continuous semantics which have been introduced to capture the notion of "sequential" continuous function. The underlying sets of the $\lambda$-models living in the stable (strongly stable) semantics are particular algebraic cpo's called dI-domains (dI-domains with coherences). These models represent all stable (strongly stable) functions between such domains. A function between dI-domains is stable if it is continuous and, furthermore, commutes with "infs of compatible elements". A strongly stable function between dI-domains with coherence, is a stable function preserving coherence.

In the next proposition we show that all models living in the main semantics are simple algebras.

Proposition 6. All $\lambda$-models living in Scott, stable and strongly stable semantics are simple algebras.

Proof. Let $\phi$ be a congruence on a Scott model $\mathbf{A}$ and let $a \phi b$ with $a \neq b$ and $a \not \leq b$. Since the continuous function $g_{c}$, defined by $g_{c}(x)=$ if $x \not \leq b$ then $c$ else $\perp$, is representable in the model (for all $c$ ), we have: $c=g_{c}(a) \phi g_{c}(b)=\perp$, hence $c \phi \perp$ for all $c$. By the arbitrariness of $c$ we get that $\phi$ is trivial.

Suppose now that $\mathbf{A}$ is a (strongly) stable model. Consider two elements $a, b \in A$ such that $a \phi b, a \neq b$ and $a \not \leq b$. There is a compact $d$ such that $d \leq a$ and $d \not \leq b$. The step function $f_{d, c}$, defined by : $f_{d, c}(x)=$ if $d \leq x$ then $c$ else $\perp$, is (strongly) stable for every element $c$. Then $c=f_{d, c}(a) \phi f_{d, c}(b)=\perp$.

\subsection{Incompleteness}

We now remark that the class of directly indecomposable combinatory algebras is a universal class.

To simplify the notation, in the following we write the combinators as $\lambda$ terms. For $\lambda$-terms $t, u$, we define the pair $[t, u] \equiv \lambda z$.ztu and, for every sequence we define $\left[t_{1}, \ldots, t_{n}\right] \equiv\left[t_{1},\left[t_{2}, \ldots, t_{n}\right]\right]$. Consider the following $\lambda$-terms:

$-P \equiv \lambda e \cdot[\lambda x . e x x, \lambda x y z \cdot e(e x y) z, \lambda x y z . e x z, \lambda x y z u . e(x y)(z u), e(\lambda x y . x)(\lambda x y . y)] ;$

$-Q \equiv \lambda e .[\lambda x . x, \lambda x y z . e x z, \lambda x y z . e x(e y z), \lambda x y z u . e x z(e y u), e]$.

We have that $e$ is central in a combinatory algebra if, and only if, the equation $P e=Q e$ holds.

Proposition 7. The class of all directly indecomposable combinatory algebras is a universal class. 
Proof. The class of all directly indecomposable combinatory algebras is axiomatised by the following universal formula:

$$
\forall e((P e=Q e \rightarrow e=\lambda x y \cdot x \vee e=\lambda x y \cdot y) \wedge \neg(\lambda x y \cdot x=\lambda x y \cdot y)) .
$$

Corollary 1. Let $\mathbf{A}$ be a combinatory algebra. Then $\mathbf{A}$ is isomorphic to a weak Boolean product of directly indecomposable combinatory algebras.

Proof. By Proposition 7 and Theorem 4.

We are now ready to provide the algebraic incompleteness theorem. We recall that a class $\mathcal{C}$ of models of $\lambda$-calculus is incomplete if there exists a consistent $\lambda$-theory $T$ such that $T \neq T h(\mathcal{M})$ for every $\mathcal{M} \in \mathcal{C}$.

Theorem 5. The indecomposable semantics is incomplete.

Proof. $\Omega \equiv(\lambda x . x x)(\lambda x . x x)$ can be consistently equated to every closed term. Let $T_{1}$ be the $\lambda$-theory generated by $\Omega=\lambda x y . x$ and $T_{2}$ be the $\lambda$-theory generated by $\Omega=\lambda x y$. Then $\Omega$ is central in the term model of $T_{1} \cap T_{2}$.

$\Omega$ is central in a combinatory algebra $\mathbf{A}$ if, and only if, $\mathbf{A}=P \Omega=Q \Omega$, where $P$ and $Q$ are the closed terms defined at the beginning of this section. Let $\mathcal{M}$ be a $\lambda$-model such that $T h(\mathcal{M})=T_{1} \cap T_{2}$. Then $\mathcal{M} \models P \Omega=Q \Omega$, because the identity $P \Omega=Q \Omega$ belongs to $T_{1} \cap T_{2}$. Then $\Omega$ is a non-trivial central element of $\mathcal{M}$.

Corollary 2. Scott, stable and strongly stable semantics are incomplete.

\section{The order-incompleteness problem}

The models of $\lambda$-calculus living in Scott, stable and strongly stable semantics are non-trivially ordered with a bottom element. However, it is also known that there are some models of the lambda calculus that cannot be non-trivially ordered (see $[24,26,30])$. In general, we define a combinatory algebra $\mathbf{A}$ to be unorderable if there does not exist a non-trivial partial order on $A$ for which the application operation is monotone. Of course, an unorderable model can still arise from an order-theoretic construction, for instance as a subalgebra of some orderable model. The most interesting result has been obtained by Selinger [30], who, enough surprising, has shown the following result.

Theorem 6. The term models of $\lambda \beta$ and $\lambda \beta \eta$ are unorderable.

It follows that, if $\lambda \beta$ or $\lambda \beta \eta$ is the theory of a po-model, then the denotations of closed terms in that model are pairwise incomparable, i.e. the term denotations form an anti-chain.

Selinger's result can be used to show that the theory of a certain po-model $\mathcal{M}$ is not $\lambda \beta$ (or $\lambda \beta \eta$ ). It is sufficient to find out two closed $\lambda$-terms, whose denotations in the model $\mathcal{M}$ are related by the partial ordering. This technique has been successfully applied to some classes of models living in Scott continuous 
semantics. Bucciarelli-Salibra [8] have shown that $\lambda \beta$ cannot be the theory of a graph model, and Carraro-Salibra [10] have shown that $\lambda \beta \eta$ cannot be the theory of a reflexive Scott domain. Other results of this type can be found in Berline et al. [4].

The problem of unorderability led Selinger [30] to study the related question of absolute unorderability: a model is absolutely unorderable if it cannot be embedded in an orderable one. Plotkin conjectures in [24] that an absolutely unorderable combinatory algebra exists, but the question is still open whether this is so. Selinger has given in [30] a syntactic characterisation of the absolutely unorderable algebras in any variety of algebras in terms of the existence of a family of Mal'cev operators. Plotkin's conjecture is thus reduced to the question whether Mal'cev operators are consistent with the lambda calculus or combinatory logic.

Hereafter, we review Selinger's characterisation of absolutely unorderability.

Let $\mathbf{A}$ be an algebra of some variety $\mathcal{V}$. A preorder $\leq$ on $\mathbf{A}$ is compatible if it is monotone in each coordinate of every function symbol of $\mathcal{V}$. Then we have: (i) $\mathbf{A}$ is unorderable if it admits only equality as a compatible partial order; (ii) $\mathbf{A}$ is absolutely unorderable if, for every algebra $\mathbf{B} \in \mathcal{V}$ and every embedding $f: \mathbf{A} \rightarrow \mathbf{B}$, the algebra $\mathbf{B}$ is unorderable.

Let $\mathcal{V}$ be a variety, $\mathbf{A} \in \mathcal{V}$ and $X$ be a set of indeterminates. We denote by $\mathbf{A}[X]$ the free extension of $\mathbf{A}$ in the variety $\mathcal{V}$. The algebra $\mathbf{A}[X]$ is defined up to isomorphism by the following universal mapping properties: (1) $A \cup X \subseteq A[X]$; (2) $\mathbf{A}[X] \in \mathcal{V}$; (3) for every $\mathbf{B} \in \mathcal{V}$, homomorphism $h: \mathbf{A} \rightarrow \mathbf{B}$ and every function $f: X \rightarrow B$, there exists a unique homomorphism $f: \mathbf{A}[X] \rightarrow \mathbf{B}$ extending $h$ and $f$. When $X=\left\{x_{1}, \ldots, x_{n}\right\}$ is finite, we write $\mathbf{A}\left[x_{1}, \ldots, x_{n}\right]$ for $\mathbf{A}[X]$.

The following result by Selinger [30] characterises those algebras which are absolutely unorderable.

Theorem 7. Let $\mathcal{V}$ be a variety. An algebra $\mathbf{A} \in \mathcal{V}$ is absolutely unorderable if, and only if, there exist a natural number $n \geq 1$ and ternary terms $p_{1}, \ldots, p_{n}$ in the type of $\mathcal{V}$ such that the algebra $\mathbf{A}[x, y]$ satisfies the following identities, called (generalised) Mal'cev axioms:

$$
\begin{aligned}
x & =p_{1}(x, y, y) ; \\
p_{i}(x, x, y) & =p_{i+1}(x, y, y) \quad(i=1, \ldots, n-1) ; \\
p_{n}(x, x, y) & =y .
\end{aligned}
$$

The following result was obtained by Plotkin-Simpson for $n=1$ and by Plotkin-Selinger for $n=2$ (see [30]).

Theorem 8. For $n=1$ and $n=2$, the Mal'cev axioms are inconsistent with the lambda calculus.

Proof. We prove the theorem for $n=1$. Assume that $x=F x y y$ and Fxxy $=y$ for a $\lambda$-term $F$. Let $Y \equiv \lambda f .(\lambda x . f(x x))(\lambda x . f(x x))$ be the Curry fixpoint combinator. Then, for any $\lambda$-term $M$, define $\mu x . M \equiv Y(\lambda x . M)$. We write $\mu x_{1} \ldots x_{n} . M$ 
for $\mu x_{1} \cdot\left(\mu x_{2} \cdot\left(\cdots\left(\mu x_{n} \cdot M\right) \cdots\right)\right)$. Now let $A \equiv \mu y x . F x y z$. Then we have $A=$ $F A A z=z$ and $A=\mu x . F x A z=\mu x . F x z z=\mu x . x=\Omega$, therefore $\Omega=z$.

The question of absolute unorderability can also be formulated in terms of theories, rather than models. In this form, Selinger [30] refers to it as the orderincompleteness question.

Definition 11. A $\lambda$-theory is order-incomplete if it does not arise as the theory of a non-trivial po-model.

The problem of order-incompleteness can be also characterised in terms of connected components of a partial ordering (minimal subsets which are both upward and downward closed): a $\lambda$-theory $\mathcal{T}$ is order-incomplete if, and only if, every po-model, having $\mathcal{T}$ as equational theory, is partitioned in an infinite number of connected components, each one containing exactly one element. In other words, the partial order is the equality.

Toward an answer to the order-incompleteness problem, the author has shown the following result in [26].

Theorem 9. Every po-model $\mathcal{M}$ of the $\lambda$-theory $T$ axiomatised by the equation $\Omega x x=\Omega$ is partitioned in an infinite number of connected components, each one containing at most the denotation of one $\lambda$-term (modulo $T$ ).

The previous result has been improved in the forthcoming paper [11].

Corollary 3. The semantics of $\lambda$-calculus given in terms of po-models with a finite number of connected components is incomplete.

\section{References}

1. Barendregt H.P.: The lambda calculus: Its syntax and semantics. North-Holland Publishing Co., Amsterdam (1984)

2. Bastonero O. and X. Gouy: Strong stability and the incompleteness of stable models of $\lambda$-calculus. Annals of Pure and Applied Logic 100, 247-277 (1999)

3. Berline C.: From computation to foundations via functions and application: The $\lambda$ calculus and its webbed models. Theoretical Computer Science 249, 81-161 (2000)

4. Berline C., Manzonetto G. and Salibra A.: Effective $\lambda$-models versus recursively enumerable $\lambda$-theories. Math. Struct. in Comp. Science 19, 897-942 (2009)

5. Berry G.: Stable models of typed lambda-calculi. Proc. 5th Int. Coll. on Automata, Languages and Programming, LNCS vol.62, Springer (1978)

6. Bigelow D. and Burris S.: Boolean algebras of factor congruences. Acta Sci. Math. 54, 11-20 (1990)

7. Bucciarelli A. and Ehrhard T.: Sequentiality and strong stability. LICS'91, IEEE Computer Society Press (1991)

8. Bucciarelli A. and Salibra A.: Graph lambda theories. Math. Struct. in Comp. Science Science 18, 975-1004 (2008)

9. Burris S. and Sankappanavar H.P.: A course in universal algebra. Springer, Berlin (1981). 
10. Carraro A. and Salibra A.: Reflexive Scott Domains are Not Complete for the Extensional Lambda Calculus. LICS'09, IEEE Computer Society Press (2009)

11. Carraro A. and Salibra A.: On the equational consistency of order-theoretic models of the $\lambda$-calculus. Forthcoming (2012)

12. Church A.: A set of postulates for the foundation of logic. Annals of Math. 2, 346-366 (1933)

13. Church A.: The calculi of lambda conversion. Princeton University Press (1941)

14. Comer S.: Representations by algebras of sections over Boolean spaces. Pacific Journal of Mathematics 38, 29-38 (1971)

15. Curry H.B. and Feys R.: Combinatory Logic Vol. I. North-Holland Publishing Co. (1958)

16. Honsell F. and Ronchi della Rocca S.: An approximation theorem for topological $\lambda$-models and the topological incompleteness of $\lambda$-calculus. Journal Computer and System Science 45, 49-75 (1992)

17. Kerth R.: Isomorphisme et équivalence équationnelle entre modèles du $\lambda$-calcul. Thèse, Université de Paris 7 (1995)

18. Kerth R.: On the construction of stable models of $\lambda$-calculus. Theoretical Computer Science 269, 23-46 (2001)

19. Lusin S. and Salibra A.: The lattice of lambda theories. Journal of Logic and Computation 14, 373-394 (2004)

20. Manzonetto G. and Salibra A.: From $\lambda$-calculus to universal algebra and back. In MFCS08, LNCS 5162, Springer, 479-490 (2008)

21. Manzonetto G. and Salibra A.: Applying universal algebra to lambda calculus. Journal of Logic and Computation, 20(4), 877-915 (2010)

22. Pierce R.S.: Modules over Commutative Regular Rings. Memoirs of AMS (1967)

23. Pigozzi D. and Salibra A.: Lambda abstraction algebras: representation theorems. Theoretical Computer Science 140, 5-52 (1995)

24. Plotkin G.D., On a question of H. Friedman. Information and Computation 126, 74-77 (1996)

25. Salibra A.: On the algebraic models of lambda calculus. Theoretical Computer Science 249, 197-240 (2000)

26. Salibra A.: Topological incompleteness and order incompleteness of the lambda calculus. ACM TOCL 4(3) (2003)

27. Salibra A., Ledda A., Paoli F. and Kowalski T. : Boolean-like algebras. Algebra Universalis (to appear)

28. Scott D.S.: Models for the $\lambda$-calculus. In Toposes, Algebraic Geometry and Logic. LNM 274, Springer (1972)

29. Scott D.S., Lambda calculus: some models, some philosophy. The Kleene Symposium, North- Holland (1980)

30. Selinger P.: Order-incompleteness and finite lambda reduction models. Theoretical Computer Science 309, 43-63 (2003)

31. Vaggione D.: Varieties in which the Pierce stalks are directly indecomposable. Journal of Algebra 184, 424-434 (1996) 